\title{
THE STABILITY OF DISPOSAL ORBITS AT SUPER-SYNCHRONOUS ALTITUDES
}

\author{
H.G. Lewis \\ G.G. Swinerd \\ University of Southampton, Southampton UK \\ hglewis | ggs@ soton.ac.uk \\ C.E. Martin \\ QinetiQ, Farnborough, UK \\ CEMartin@ space.QinetiQ.com

\section{W.S. Campbell} \\ The Aerospace Corporation, Albuquerque NM, USA \\ WSpencer.Campbell@aero.org
}

\begin{abstract}
An investigation of the stability of super-synchronous disposal orbits using University of Southampton's DAMAGE (Debris Analysis and Monitoring Architecture for the Geosynchronous Environment) is presented. The DAMAGE orbital propagator was used to analyse the influence of the initial perigee, eccentricity, right ascension of ascending node (RAAN) and argument of perigee, and the initial lunar RAAN, on the evolution of disposal orbit perigee over a 200-year period. This propagator includes perturbations arising from low-order gravitational harmonics, third-body influences and solar radiation pressure (SRP). The results of two sensitivity studies are presented. The first evolved 14,112 disposal orbits with initial perigees at the minimum altitude suggested by the IADC guideline, whilst the second study evolved 17,920 disposal orbits with initial perigees from $42,374 \mathrm{~km}$ to 42,464 $\mathrm{km}$. The studies show that the initial perigee and eccentricity of the disposal orbit are the most important factors for maintaining the orbit above the protected region. Some sensitivity to the initial lunar RAAN, disposal orbit RAAN and argument of perigee was also found. These findings suggest that the IADC guideline specifying a preferred initial perigee is appropriate if it accompanies a guideline for the initial eccentricity of the disposal orbit.
\end{abstract}

Copyright (C) 2003 by the International Astronautical Federation. All rights reserved.

\section{INTRODUCTION}

To reduce the effect of dead satellites on the operational population, the Inter-Agency Space Debris Coordination Committee (IADC) has specified a guideline for the minimum perigee of geosynchronous disposal orbits for satellites at endof-life. To account for third-body (lunisolar) gravitational effects, the IADC guideline specifies a graveyard orbit with a minimum perigee altitude 35 $\mathrm{km}$ above a manoeuvre corridor (also referred to as the protected region) that extends $200 \mathrm{~km}$ above the synchronous altitude of $35,786 \mathrm{~km}$ (this altitude is referred to as GEO throughout). Additionally, orbits at this altitude are also influenced by solar radiation pressure (SRP) and, consequently, the IADC guideline for minimum perigee contains allowances for perturbations induced by SRP as well, as shown in Figure 1. In this Figure the operational region is defined as GEO $\pm 35 \mathrm{~km}$. The IADC guideline for minimum perigee is

$$
\Delta H=235+1000 C_{r}\left(\frac{A}{M}\right)
$$

where $\Delta H$ is the new perigee altitude above GEO, $A$ is the satellite average cross-sectional area, $M$ is the satellite mass and $C_{r}$ is the radiation pressure coefficient.

Debris environment models have been used to investigate the effect of this mitigation approach on the long-term collision risk in the geostationary ring ${ }^{1}$. In addition, studies by Chobotov ${ }^{2}$, $\mathrm{Chao}^{3}$, Lewis et $a$. $^{4}$ and others have investigated the longterm stability of super-synchronous disposal orbits 
using a variety of propagation techniques and sensitivity studies.

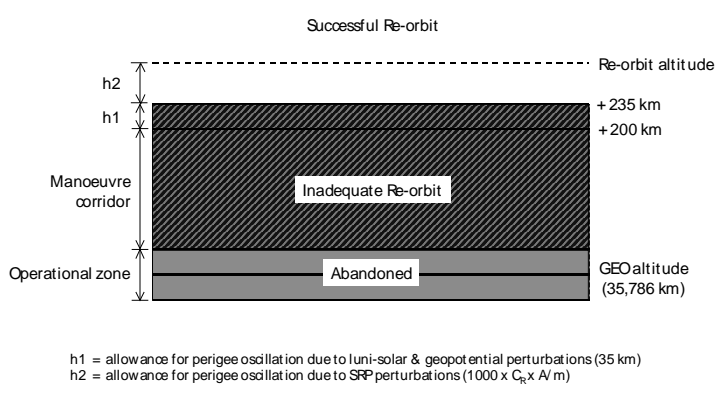

Figure 1. Schematic showing re-orbiting regions corresponding to the current IADC guideline.

Chobotov $^{2}$ carried out long-term numerical integration (up to 42 years) and found the $1^{\text {st }}$-order, nearly-resonant perturbation for circular orbits at GEO $+150 \mathrm{~km}$ to result in a maximum radial excursion of about $2.31 \mathrm{~km}$, due almost entirely to the lowest order tesseral harmonic $\mathbf{J}_{2,2}$. In-plane motion results mostly from solar radiation pressure. However, perturbations in eccentricity due to lunisolar gravitational attractions were not considered. Chobotov concluded that the long-term stability of super-synchronous orbits was adequate for disposal purposes.

$\mathrm{Chao}^{3}$ identified three key objectives of his approach: first to understand the long-term variations in semi-major axis and eccentricity through analytical expansions and approximations, second to perform long-term integration with both numerical and semianalytical propagators of super-synchronous orbits up to 100 years, and third to base his conclusions on the results of both the analytic expressions and the 100year propagations. Examination of the analytic expansions showed that long-term eccentricity variations caused by lunisolar perturbations might have significant amplitude; also, variations in eccentricity might be dependent on epoch, initial eccentricity, RAAN and argument of perigee. Two independent orbit propagation tools were used for the numerical integration: one based on the highprecision semi-analytic orbit propagator developed by Draper Laboratory and the other using Cowell's method with automatic error control in the $n$-body integration of satellite orbits. Chao used the following force models and initial conditions:

\section{Perturbing forces:}

- 8 by 8 WGS 84 Earth gravity model

- Sun/Moon gravitational attractions

- Solar radiation pressure
Initial orbit conditions:

- Two epochs: 18 October 1998 and 1 January 2004

- Five orbit altitudes: GEO+50 km, GEO+100 $\mathrm{km}, \mathrm{GEO}+150 \mathrm{~km}, \mathrm{GEO}+250 \mathrm{~km}$, and $\mathrm{GEO}+350 \mathrm{~km}$

- Three initial eccentricities: $0.00065,0.0035$, and 0.0065

- Initial argument of perigee: $0,90,180$, and 252.223 degrees

- Initial RAAN: 90, 180, and 279.999 degrees

- Initial inclination: 2.66950 and 4.66950 degrees

- Four surface areas for the same spacecraft mass of $2000 \mathrm{~kg}: 186 \mathrm{~m}^{2}, 18.6 \mathrm{~m}^{2}, 9.3 \mathrm{~m}^{2}$, and $4.6 \mathrm{~m}^{2}[\mathrm{~A} / \mathrm{m}=0.093,0.0093,0.00465$, and $0.0023 \mathrm{~m}^{2} / \mathrm{kg}$ ]

Chao concluded that the disposal orbits were stable; both analytical and numerical results showed that long-term eccentricity variations are wellbehaved sinusoids with no secular changes. The amplitude of the sinusoidal variations is proportional to initial eccentricity and has some dependence on initial argument of perigee, RAAN and epoch. Ten to 12 year periodic eccentricity variations are the result of Sun/Moon gravitational attractions, while annual variations in eccentricity are due to solar radiation pressure and have an amplitude that depends on areato-mass ratio.

Lewis et $a l .^{4}$ used the DAMAGE propagator to investigate the effect of initial perigee and eccentricity on the long-term (500 years) stability of super-synchronous disposal orbits. This study established a dependence on initial eccentricity and found that even for disposal orbits with an initial perigee determined using the IADC guideline the long-term stability was uncertain.

The role of mitigation in controlling the growth of the debris environment in the geosynchronous orbital region was also investigated by Martin et al. ${ }^{5}$ in a long-term evolution of the GEO population using DELTA. This work demonstrated that the removal of mass from the operational region using re-orbit manoeuvres was the optimal mitigation method available at geostationary altitudes. Additionally, Martin et al. found that the orbits of reorbited objects should be kept as near to circular as possible. In their study, increasing the eccentricity of disposal orbits caused them to evolve into the operational GEO region.

Following this previous work, we investigate the suitability of the IADC disposal orbit guideline and present a new, high resolution analysis of disposal orbit scenarios using the DAMAGE propagator. Two sensitivity studies, each varying 
initial values of eccentricity, RAAN, argument of perigee and lunar RAAN (which is dependent upon epoch), were performed in order to gain a better understanding of their influence on the evolution of perigee.

\section{LONG-TERM ORBITAL PROPAGATION}

The University of Southampton's DAMAGE model is a long-term space debris environment model valid for Earth orbits between $120 \mathrm{~km}$ and super-GEO $(\mathrm{GEO}+2,000 \mathrm{~km})$ altitudes. Rather than being an extension of an existing low Earth orbit model, DAMAGE is a new analysis tool dedicated to overcoming the challenges of modelling the GEO environment.

DAMAGE incorporates a semi-analytical orbital propagator that determines the rates of change in the classical orbital elements $a, e, i, \omega, \Omega$ arising from perturbations induced by the Earth gravitational harmonics ${ }^{6}, \mathrm{SRP}^{7}$ and lunisolar gravitational attraction $^{8}$. Of particular interest to this study were changes in semi-major axis and eccentricity, as these values define the perigee.

The resonant part of the $J_{2,2}$ harmonic produces an oscillation in semi-major axis for nearsynchronous orbits ${ }^{6}$,

$$
\begin{aligned}
\frac{d a}{d t}=- & 3 n a J_{2,2}\left(\frac{R}{a}\right)^{2}(1+\cos i)^{2}\left(1-\frac{5}{2} e^{2}+\frac{13}{16} e^{4}\right) \\
& \times \sin 2\left(\omega+M+\Omega-\lambda_{2,2}-g\right)
\end{aligned}
$$

where $R$ is the mean equatorial radius of the Earth, $\lambda_{2,2}$ is the stable longitude, $J_{2,2}$ is the constant associated with the 2,2 sectorial harmonic and $g$ is the Greenwich sidereal angle. $\mathrm{Chao}^{3}$ reported a 900day variation in semi-major axis of a GEO satellite, with an amplitude of about $30 \mathrm{~km}$ induced by resonant effects of the tesseral harmonics. In contrast, semi-major axis variations for a GEO+350 $\mathrm{km}$ orbit were reported to be $0.7 \mathrm{~km}$ due to the shallower resonant effects. Whilst the $J_{2,2}$ harmonic was modelled in the sensitivity study presented here, it was not expected to have a significant impact on the perigee variation of disposal orbits, in line with $\mathrm{Chao}^{3}$.

As mentioned previously, the main effect of SRP is to induce an annual oscillation in eccentricity ${ }^{7}$,

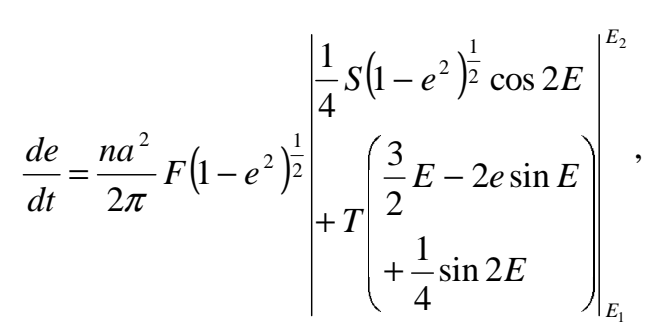

where $S$ and $T$ are direction cosines of the radiation pressure force, $F$, per unit mass along the satellite's radius vector and perpendicular to the radius vector in the orbital plane respectively. The spacecraft's moves in sunlight from eccentric anomaly $E_{1}$ to $E_{2}$ with mean motion, $n$. Chobotov ${ }^{2}$ noted the maximum cyclical perigee variation was about $24 \mathrm{~km} / \mathrm{yr}$ for a spacecraft with $A / M$ of $0.0136 \mathrm{~m}^{2} / \mathrm{kg}$, eccentricity of 0.001 , RAAN of 222 degrees and coefficient of reflection of 0.9 .

Lunisolar gravitational attraction also causes significant changes in eccentricity,

$$
\frac{d e}{d t}=\frac{15 K e\left(1-e^{2}\right)^{\frac{1}{2}}}{2 n}\left[\begin{array}{l}
A B \cos 2 \omega \\
-\frac{1}{2}\left(A^{2}-B^{2}\right) \sin 2 \omega
\end{array}\right],
$$

where $K$ is a gravitational constant of the disturbing body, and $A$, and $B$ are the direction cosines of the radius vector to the disturbing body referred to the geocentric axes through the ascending node of the satellite, through the apex of the orbit and normal to the orbit respectively ${ }^{8}$.

QinetiQ's Debris Environment Long-Term Orbit Propagator (DELTOP) is also a semi-analytical propagator, and, with the exception of perturbations due to tesseral harmonics, the rates of change of orbital elements are determined using the same theoretical sources as the DAMAGE propagator. A comparison of the perigee-history of six disposal orbits evolved by DAMAGE and DELTOP indicated good agreement between the two propagators. Figure 2 shows the perigee history for a disposal orbit with initial parameters as in Table 1.

Table 1. Initial parameters of disposal orbit used for propagator validation

\begin{tabular}{|ll|}
\hline$a$ & $42464.12 \mathrm{~km}$ \\
$e$ & 0.005 \\
$i$ & 0.04 degrees \\
$\omega$ & 60 degrees \\
$\Omega$ & 0 degrees \\
$A / M$ & $0.01 \mathrm{~m}^{2} / \mathrm{kg}$ \\
$C_{R}$ & 1.2 \\
\hline
\end{tabular}




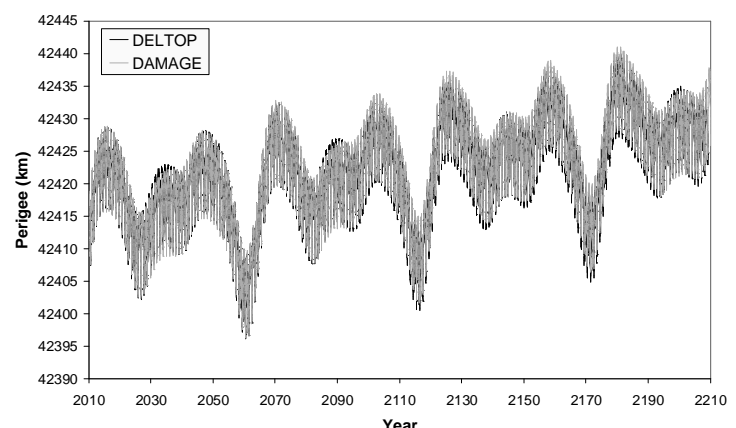

Figure 2. Two hundred-year orbital propagator comparison.

QinetiQ's Orbit Software Suite (OSS) consists of a number of orbit propagators and utility routines that have been developed over the last quarter century to help control the UK's Skynet series of geosynchronous satellites. It has been validated operationally over this period and is used on a daily basis for outputs such as antenna pointing angles, burn prediction and eclipse prediction. The OSS employs a multi-step integration algorithm (8th order Gauss Jackson), has an operational accuracy of metres and models the following perturbations:

- Geopotential (to 5th order)

- Earth axis precession

- Lunar and solar gravity

- Solar radiation pressure (including Earth shadow effects)

- Atmospheric drag.

The OSS provided an important benchmark for the DAMAGE propagator used in this sensitivity study. The thirty-year perigee history of the disposal orbit in Table 1 produced by the OSS is compared with the perigee histories produced by DAMAGE and DELTOP in Figure 3.

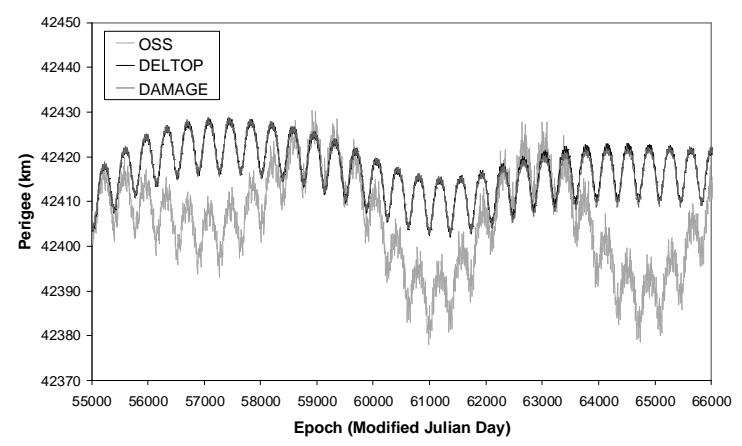

Figure 3. Thirty-year orbital propagator comparison

Figure 3 shows with greater resolution the good agreement between DELTOP and DAMAGE.
All three propagators capture the amplitude and frequency of the annual variation caused by SRP with only small, high frequency differences being apparent. However, the long-period changes due to lunisolar gravitational attraction predicted by the OSS are different to those predicted by DAMAGE and DELTOP. Figure 3 shows a $50 \mathrm{~km}$ amplitude, $\sim 15$ year cycle in perigee captured by the OSS that is not present in the DAMAGE/DELTOP perigeehistory. Chobotov ${ }^{2}$ recorded a $\sim 10$ year oscillation in eccentricity that possibly corresponds with the OSSpredicted oscillation. The difference between DAMAGE/DELTOP and the OSS was assumed to arise from the inclusion of higher order terms for lunisolar perturbations in OSS. However, investigations are continuing.

\section{DISPOSAL ORBIT SENSITIVITY STUDIES}

\section{$\underline{\text { Disposal orbit characteristics }}$}

Two sensitivity studies were performed. The first evolved 14,112 disposal orbits with initial perigees at the minimum altitude suggested by the IADC guideline (1), whilst the second study evolved 17,920 disposal orbits with initial perigees from $42,374 \mathrm{~km}$ to $42,464 \mathrm{~km}$.

Previous work has predicted that the maintenance of disposal orbit perigees above the manoeuvre corridor (protected region) is dependent on the initial eccentricity, RAAN, argument of perigee and epoch. The dependence on epoch is primarily due to the variation in the right ascension of the ascending node of the moon, $\Omega_{M}$, which oscillates between approximately -13 and +13 degrees with a period of about 18.6 years. Figure 4 shows the change in lunar RAAN versus epoch as an output from QinetiQ's OSS ephemeris. The conversion from lunar RAAN to epoch was made using this data.

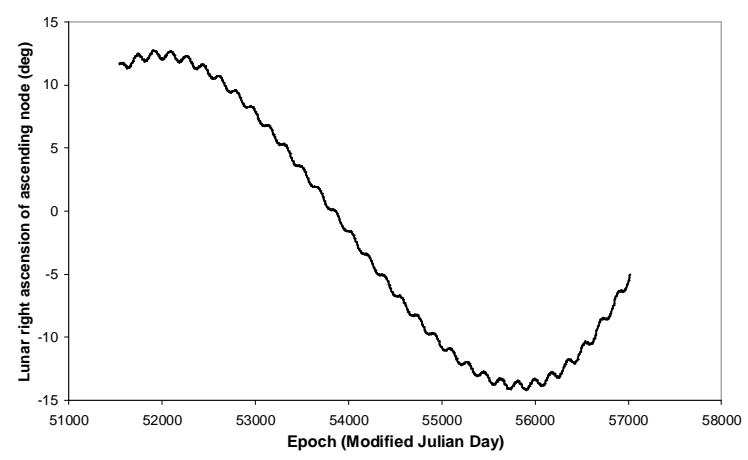

Figure 4. Variation in lunar right ascension of ascending node versus epoch (output from QinetiQ's OSS). 
The range of eccentricity values of satellites currently in GEO and GEO disposal orbits (GEO + $250 \mathrm{~km}$ ) was determined using DISCOS data and is shown in Figure 5 (epoch: 1st September 2003). The sensitivity studies used a broad range of eccentricity values that incorporated the current population's range at the lower end in an effort to gain a better understanding of the influence of higher eccentricity values on the perigee evolution of disposal orbits.

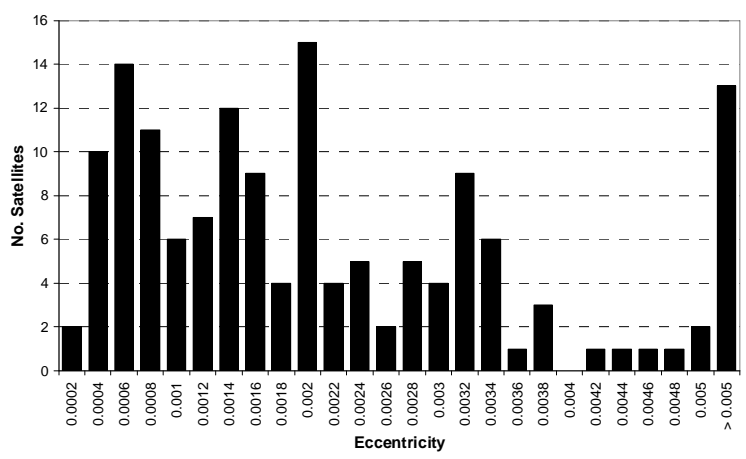

Figure 5. Distribution of eccentricity for satellites in GEO disposal orbits (data from DISCOS).

Values of RAAN and argument of perigee were chosen at regular intervals in the range $[0,360]$ degrees.

Both sensitivity studies varied initial eccentricity, RAAN, argument of perigee and epoch, with the second study also varying initial perigee. Table 2 details the values used for each variable parameter in the first sensitivity study and Table 3 has the corresponding values for the second sensitivity study.

Table 2. Variable parameter values used in first sensitivity study

\begin{tabular}{|ll|}
\hline$e$ & $0.0001,0.0005,0.001,0.005,0.01,0.05,0.1$ \\
$\omega$ & $0-330$ degrees in 30 degree steps \\
$\Omega$ & $0-330$ degrees in 30 degree steps \\
$\Omega_{M}$ & $-14-+12$ degrees in 2 degree steps \\
\hline
\end{tabular}

Table 3. Variable parameter values used in second sensitivity study

\begin{tabular}{|ll|}
\hline perigee & $42,374-42,464 \mathrm{~km}$ in $10 \mathrm{~km}$ steps \\
$e$ & $0.0001,0.001,0.01,0.1$ \\
$\omega$ & $0-315$ degrees in 45 degree steps \\
$\Omega$ & $0-315$ degrees in 45 degree steps \\
$\Omega_{M}$ & $-12-+12$ degrees in 4 degree steps \\
\hline
\end{tabular}

Reading from Tables 2 and 3 it can be seen that the first sensitivity study comprised 14,112 disposal orbits $(7$ eccentricities $\times 12$ RAAN $\times 12$ arguments of perigee $\times 14$ epochs) and the second study comprised 17,920 disposal orbits (4 eccentricities $\times 8$ RAAN $\times 8$ arguments of perigee $\times$ 7 epochs $\times 10$ perigees).

The initial values of the remaining orbital elements and satellite properties were fixed for all orbits. Appropriate values of $A / M$ were determined from the satellites currently in GEO and GEO disposal orbits using DISCOS data again. The mean area-to-mass ratio for this current population was found to be approximately $0.01 \mathrm{~m}^{2} / \mathrm{kg}$. The distribution of $A / M$ for satellites with perigees greater than GEO $+250 \mathrm{~km}$ is shown in Figure 6.

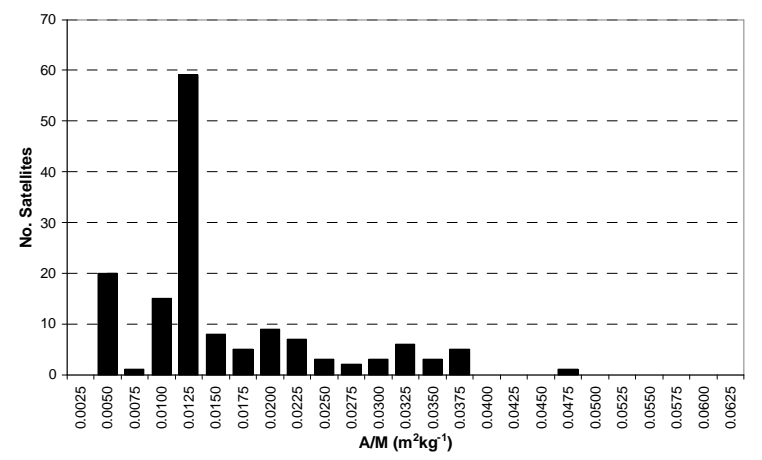

Figure 6. Distribution of area-to-mass for satellites in GEO disposal orbits (data from DISCOS).

The initial inclination of the disposal orbits was assumed to be near to the North-South deadband limit for a geostationary satellite (0.05 degrees), as would be the case for an end-of-life disposal scenario. In addition, the reflectivity coefficient, $C_{R}$, was assumed to have a value of 1.2 , which is the value commonly used within debris environment models such as DAMAGE. Table 4 details the values used for the fixed parameters in both sensitivity studies.

Table 4. Fixed parameter values used in sensitivity studies

\begin{tabular}{|ll|}
\hline Perigee & $42,411 \mathrm{~km}$ (study 1 only) \\
$i$ & 0.04 degrees \\
$A / M$ & $0.01 \mathrm{~m}^{2} / \mathrm{kg}$ \\
$C_{R}$ & 1.2 \\
\hline
\end{tabular}

The fixed and variable initial conditions were used to generate two launch traffic databases suitable for DAMAGE. DAMAGE then "launched" each satellite in the database as the simulation reached epochs corresponding to the lunar RAAN values detailed in Tables 2 and 3. 


\section{$\underline{\text { Simulation characteristics }}$}

The DAMAGE propagator was used to evolve the disposal orbits from an initial Modified Julian Day (MJD) of 52096 (6th July 2001) to an end MJD of 128954 (9th December 2211). The lunar RAAN ephemeris from QinetiQ's OSS (Figure 4) was used to establish these conditions, such that the initial epoch corresponded to a lunar RAAN of 12 degrees and the end epoch was set to be 200 years later than the epoch at which the lunar RAAN was next at -14 degrees (MJD 55904, or 9th December 2011). The simulation included secular $J_{2}$, long-period $J_{3}$ and resonant $J_{2,2}$ gravitational harmonic perturbations, lunisolar perturbations and SRP effects as described above.

Information about each disposal orbit in both sensitivity studies was captured using seven parameters throughout the simulation and these are detailed in Table 5. The protected region and operational region flags were set for a particular disposal orbit if the perigee decreased below GEO + $200 \mathrm{~km}$ and GEO + $35 \mathrm{~km}$, respectively. The duration spent within the protected and operational regions represented the accumulated time for which the perigee was below GEO + $200 \mathrm{~km}$ and GEO + 35 $\mathrm{km}$, respectively.

Table 5. Disposal orbit characteristics captured during simulation

\section{Protected region flag}

Operational region flag

Duration within protected region

Duration within operational region

Time of first entry into protected region

Time of first entry into operational region

Minimum perigee achieved during simulation

\section{$\underline{\text { RESULTS }}$}

\section{$\underline{\text { Initial remarks }}$}

The two sensitivity studies were performed on a desktop PC with an AMD 2600+ processor running the DAMAGE software. The run-times for the sensitivity studies were around 60 minutes.

The results below are categorised into general findings, obtained from the last three characteristics listed in Table 5, and specific findings that identify the dependence of the protected region and operational region flags on the initial orbital conditions. The results of both sensitivity studies have been normalised to allow for easy comparison. This normalisation simply converts from the number of satellites to the proportion of satellites with a particular property. The results originate from the first sensitivity study, unless indicated otherwise.

\section{$\underline{\text { General characteristics }}$}

Figure 7 indicates that the majority of disposal orbits in study 1 , approximately $75 \%$, remained above the protected region throughout the 200-year simulation. Additionally, about $85 \%$ remained above the operational region. Figure 7 also shows that the minimum perigees of approximately $60 \%$ of the disposal orbits remained within a few kilometres of their initial perigee.

Figure 8 shows that approximately $16 \%$ of the satellites in study 1 entered the protected region within 10 years with a further $7 \%$ following within 20 years. That is, nearly all satellites that entered the protected region did so within 20 years. The trend is similar for satellites that entered the operational region; most did so within 20 years.

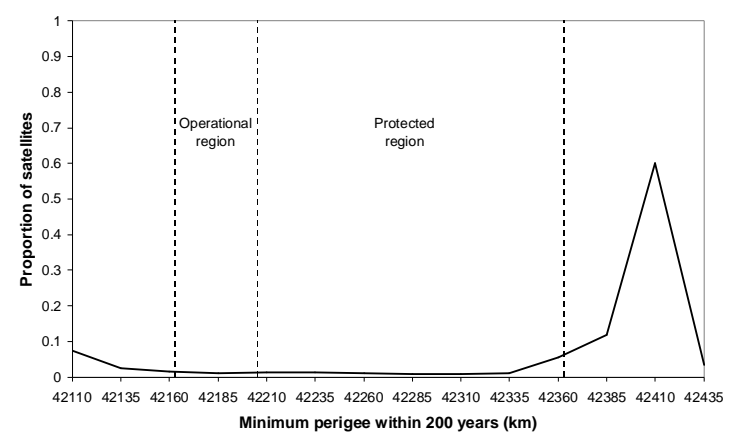

Figure 7. Distribution of minimum perigee.

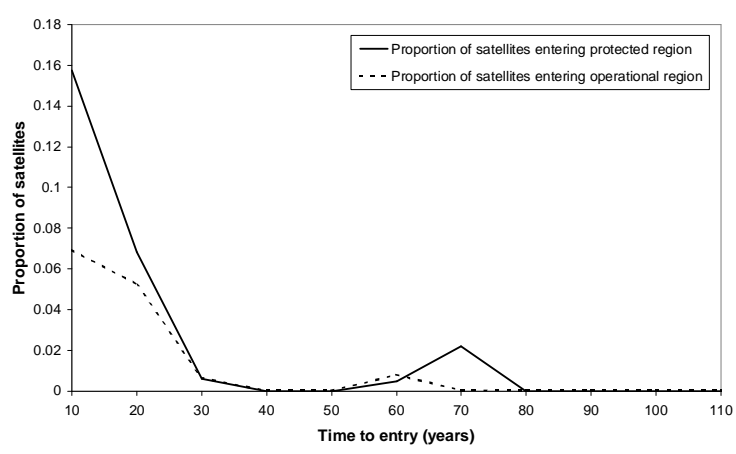

Figure 8. Proportion of satellites entering the protected/operational regions as a function of entry time. 


\section{$\underline{\text { Sensitivity to initial conditions }}$}

Figure 9 highlights the apparent dependency of the protected/operational region flags on the initial perigee of the disposal orbit. The curve shown in the Figure shows an exponential increase in the proportion of satellites entering the protected region as the initial perigee decreases. The Figure shows that approximately $75 \%$ of satellites in initial disposal orbits with perigees just above the protected region $(42,374 \mathrm{~km})$ entered this region.

Figure 9 also indicates that increasing the initial disposal orbits perigees above $42,411 \mathrm{~km}$ (the IADC guideline for satellites in this study) only offered a small (5\%) improvement in terms of the proportion of satellites entering the protected and operational regions. This is because the IADC guideline puts the initial perigee at the lower part of the exponential curve in Figure 9. Further, it is also evident that initial perigee was not a significant influence on whether the satellite entered the operational region.

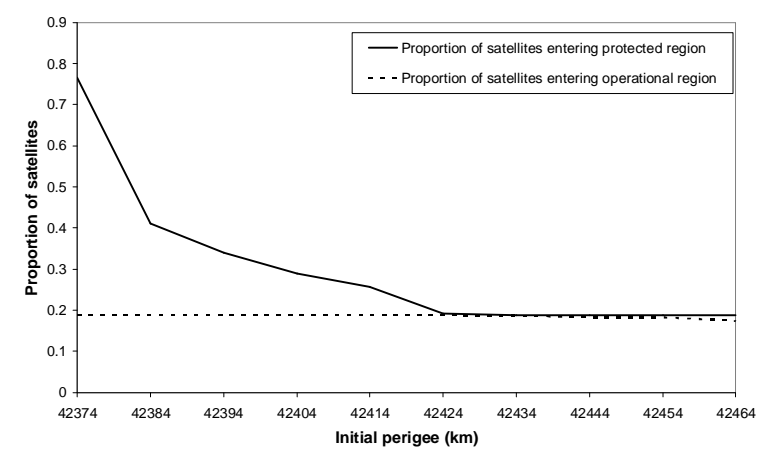

Figure 9. Proportion of satellites entering the protected/operational regions as a function of initial perigee from study 2 .

Figure 10 indicates a significant sensitivity to the initial eccentricity of the disposal orbit. Orbits with eccentricities less than 0.005 did not enter the protected region. Similarly, orbits with eccentricities less than 0.01 did not enter the operational region. This finding confirms the conclusions reported in Lewis et al. ${ }^{4}$ and Martin et al. ${ }^{5}$ which suggested that disposal orbits should be circularised.

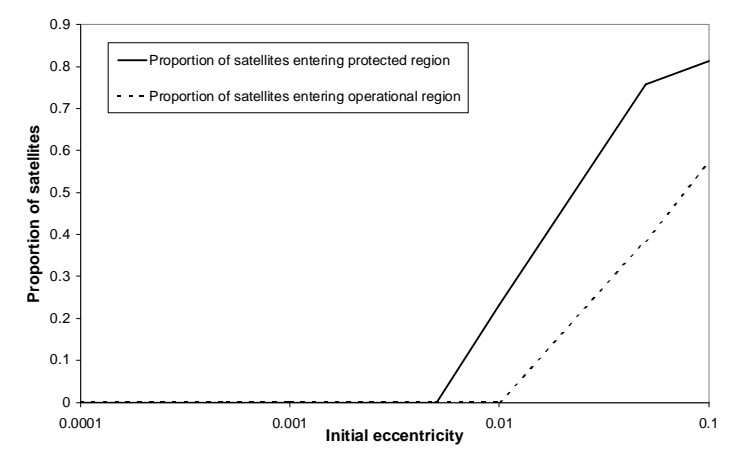

Figure 10. Proportion of satellites entering the protected/operational regions as a function of initial eccentricity.

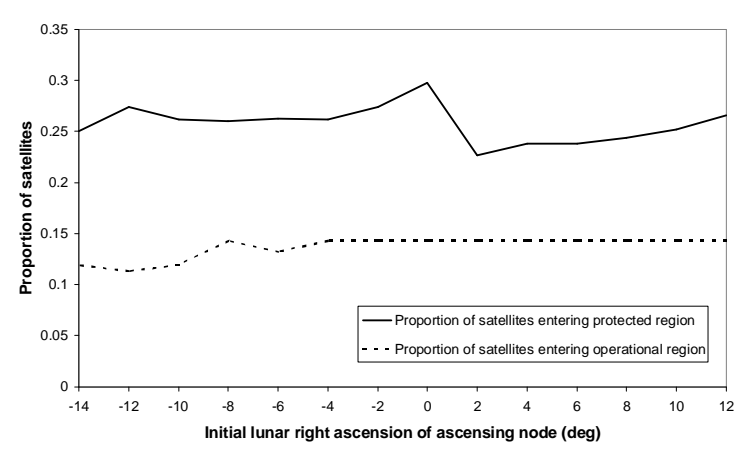

Figure 11. Proportion of satellites entering the protected/operational regions as a function of initial lunar RAAN.

Figure 11 displays a small effect of the initial lunar RAAN. Satellites disposed at an epoch when the lunar RAAN is zero or at \pm 12 degrees were slightly more likely $(10 \%)$ to enter the protected region than for other lunar RAAN values.

A more detailed analysis of the data indicated that lunar RAAN played a role only when it had values of +12 and zero degrees, and the initial eccentricity of the disposal orbit was 0.1 , as shown in Figure 12. It should be noted that points showing both a circle and a star in Figure 12 indicate variables other than initial lunar RAAN and initial eccentricity determine whether the disposal orbits enter the protected region. This result is of only minor importance and is provided for completeness, as earlier work has shown that circularising initial disposal orbits (i.e. low initial eccentricities) is good practice. 


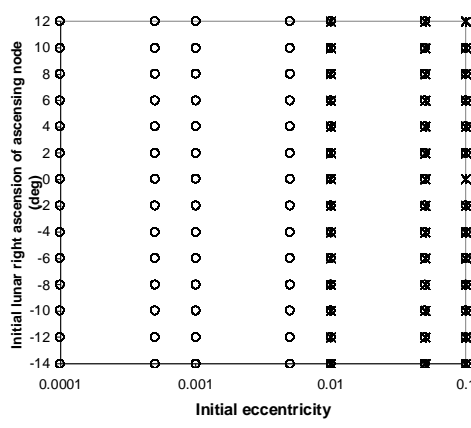

Figure 12. Distribution of initial satellite eccentricity and initial lunar RAAN for satellites above the protected region and satellites within the protected region.

Figures 13 and 14 show a small influence of initial RAAN and argument of perigee on the protected and operational region flags. The scale of these Figures has been selected to highlight the fact that these influences are minor. However, the curves in Figure 13 are sinusoids in RAAN with a maximum at 180 degrees and a minimum at zero degrees, whilst the curves in Figure 14 are sinusoids with an argument of twice the argument of perigee and maxima at zero and 180 degrees.

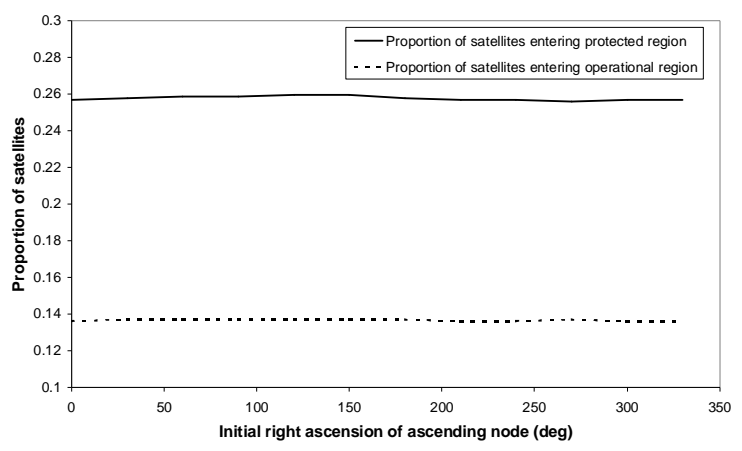

Figure 13. Proportion of satellites entering the protected/operational regions as a function of initial satellite RAAN.

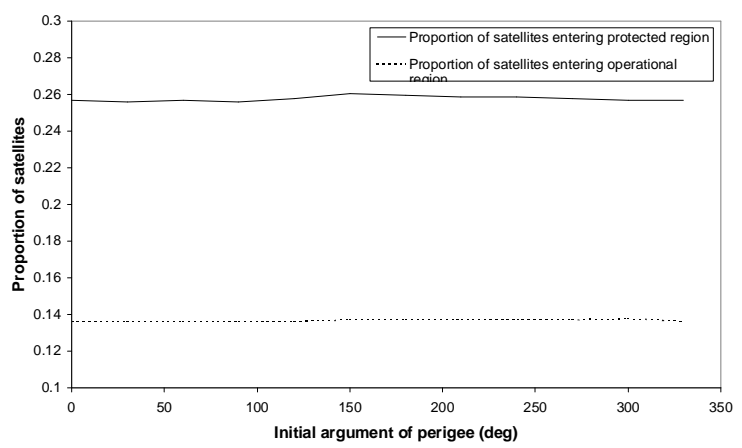

Figure 14. Proportion of satellites entering the protected/operational regions as a function of initial argument of perigee.
The sensitivity of the operational region flag to initial RAAN and argument of perigee is shown more clearly in Figure 15.

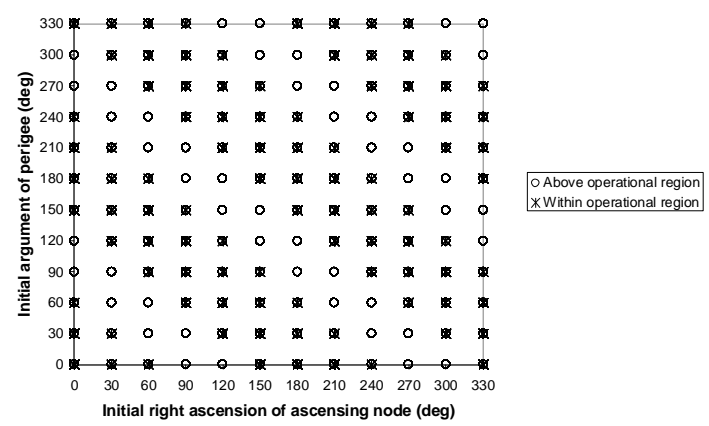

Figure 15. Distribution of initial satellite RAAN and argument of perigee for satellites above the operational region and satellites within the operational region.

Disposal orbits with initial RAANs and argument of perigee values that sum approximately to multiples of 90 degrees remained above the operational region, whereas other values of RAAN and argument of perigee resulted in the satellite entering the operational region. This result ties in with the analytic, singly averaged expression for the rate of change of eccentricity due to the major perturbations ${ }^{3}$,

$\frac{d e}{d t}=e K[0.298 \sin 2(\omega+\Omega)-0.038 \sin (2 \omega+\Omega)]$,

where $K$ is a small coefficient due to the third-body attraction, and the long-term average of the lunar RAAN is assumed to be zero.

It is important to note that the clear effect of initial RAAN and argument of perigee for satellites that entered the operational region was not apparent for satellites that entered the protected region. This may be due to more significant influences, such as the value of initial eccentricity, hiding this effect of initial RAAN and argument of perigee.

\section{CONCLUSIONS}

The suitability of the IADC guideline for disposal orbit perigees has been investigated using the DAMAGE long-term propagator. Two sensitivity studies were performed, looking specifically at the influence of initial perigee, eccentricity, RAAN, argument of perigee and epoch. Large sensitivities to the values of initial perigee and eccentricity were found, with minor sensitivity to values of RAAN, argument of perigee and epoch. 
The results suggest that the IADC guideline of GEO plus no less than $235 \mathrm{~km}$ for the perigees of disposal orbits is appropriate if the initial eccentricity of the orbit is less than 0.005 . No great benefit was gained by increasing the initial perigee, but lowering the initial perigee caused an exponential rise in the proportion of satellites that entered the protected region.

\section{$\underline{\text { ACKNOWLEDGEMENTS }}$}

The Engineering and Physical Sciences Research Council provided financial support for part of this work. Thanks to C.C. "George" Chao, Marlon Sorge (The Aerospace Corporation), Damian Smith, Duncan Smith (QinetiQ), Neil Williams, Gavin Gittins, and Sarah Stevenage (University of Southampton) for their helpful contributions.

\section{$\underline{\text { REFERENCES }}$}

[1] Pardini, C. and Anselmo, L., Influence of the Spacecraft End-of-life Re-orbiting Altitude on the Long-term Collision Risk in the Geostationary Ring. $33^{\text {rd }}$ COSPAR Scientific Assembly, Warsaw, Poland, 16-23 July 2000.

[2] Chobotov, V.A., Disposal of Spacecraft at Endof-Life in Geosynchronous Orbit. Journal of Spacecraft and Rockets, Vol.27, No. 4, JulyAugust 1990, pp. 433-437.

[3] Chao, C.C., Geosynchronous Disposal Orbit Stability. Aerospace Report No. TOR-97(1106)7, September 1997.

[4] Lewis, H.G., Swinerd, G.G., Williams, D.N, and Gittins, G.L., Investigating the long-term evolution of the debris environment in high earth orbit using the DAMAGE model, AIAA Paper No. IAA-01-IAA-6-6-01, International Astronautical Congress, Toulouse, France, October 2001

[5] Martin, C.E., Stokes, P.H., and Walker, R., The long-term evolution of the debris environment in high earth orbit including the effectiveness of mitigation measures, International Astronautical Congress, Toulouse, France, October 2001.

[6] Kaula, W.M., Theory of satellite Geodesy: applications of satellites to Geodesy, Dover Publications Inc., Mineola, New York, 2000.
[7] Aksnes, K., Short-period and long-period perturbations of a spherical satellite due to direct solar radiation. Center for Astrophysics Preprint Series No. 230, Harvard College Observatory and Smithsonian Astrophysical Observatory, Cambridge, Massachusetts, 1974.

[8] Cook, G.E., Luni-solar perturbations of the orbit of an Earth satellite. Royal Aircraft Establishment Technical Note No. G.W. 582, Ministry of Aviation, London, 1961. 